\section{Alexander Meng}

\section{Einleitung}

«Über die Diagnose, Therapie, Basistheorie der TCM, Nadelmanipulationstechnik, TENS, elektrische Nadelstimulation werden wir sprechen. (...) Besonders möchte ich hinweisen auf die gesetzlich verankerte Stellung der Akupunktur, wie sie z.B. vom Obersten Sanitätsrat nach genauer Prüfung und sehr präzisem Bescheid dargelegt wurde und heute auch als allgemein gültig, besonders von der ÖAK aus, anerkannt wird. (...) In Österreich gibt es rechtlich einen Unterschied zwischen Akupunktur und sog. TCM, aus der die Akupunktur wohl stammt.» (Zitate von Johannes Bischko in der Österreichischen Ärztezeitung, Juli 1997)

Die Medizintheorie der Traditionellen Chinesischen Medizin (TCM) 中医 basiert in erster Linie auf empirischen Erfahrungen. Im vor über 2000 Jahren publizierten, ältesten Lehrbuch "Neijing 难经》 finden wir Abhandlungen über Anatomie, Phy-

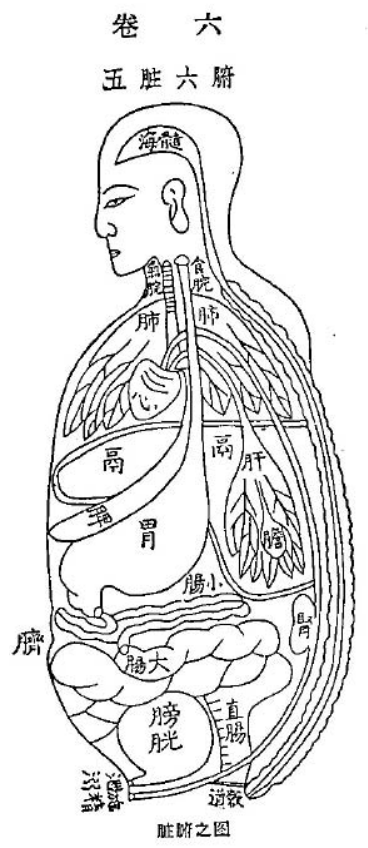

Abb. 1. Zhenjiu Dacheng 针尒大成, Yang Jizhou 杨继洲, 1601 [6]. siologie, Pathophysiologie, Ätiologie, Diagnostik, Therapie und Prophylaxe. Die Hauptthesen der TCM lauten unter anderem: die Lehre von Yin und Yang, die Lehre von den 5 Elementen und die Lehre von den Meridianen. Die moderne Medizin (西医) hat seit dem Altertum grossen Wert auf die Anatomie gelegt. Im Vergleich dazu sind die anatomischen Kenntnisse der TCM vage.

\section{Anatomie}

Von den alten Griechen und Römern über die Renaissance bis zur Gegenwart interessierte man sich besonders intensiv für die Details der Natur inklusive des Menschen. Im alten China gab es nur sporadische Angaben über die medizinischen Seziertätigkeiten, aber das Interesse der Tradition der chinesischen Kultur gilt nicht der Natur und auch nicht den Erscheinungsformen der Natur, sondern inneren Werten und inneren Zusammenhängen des Menschen mit der Natur.

Nicht die Natur des Menschen ist wichtig, sondern seine sozialethische, sittliche Bedeutung, da nur der Mensch über das Bewusstsein der Ethik verfügt. Nur der Besitz der Ethik macht die höhere Wertigkeit des Menschen in der Natur aus.

Konfuzius verachtete die Erforschung von materiellen Objekten, daher auch die Anatomie (Abb. 1). Da die Chinesen seit dem Altertum die Nützlichkeit sehr schätzen, ist es auch verständlich, dass die Grundlagenforschung als technische Perversion diskreditiert wurde.

\section{Atom/Qi}

Im Westen haben die alten Griechen das Atom als Bestandteil der Materie angesehen. Die exakte und fortwährend feinere Strukturforschung (Grundlagenforschung der Anatomie) wurde stets in Verbindung mit der Funktion gebracht. Im alten China wurde das Modell Qi als Bestandteil des Universums und des Menschen gesehen.

\section{Strukturelle Funktion/ \\ Sinnerfassung}

In der modernen Medizin bedarf jede Funktion einer strukturellen Untermauerung. Saloppe Formulierung: Ist die moderne Medizin eine «Reparaturmedizin»?

Die TCM legt höchsten Wert auf die Sinnerfassung (Deyi 得意), sogar auf Phänomene, wobei auf Erscheinungsbilder verzichtet wird (Wangxiang 忘象). In der Kunst wird nicht auf die Abbildung der Wirklichkeit, der Natur, Wert gelegt. Das Wichtigste an einem Kunstwerk ist der geistige Ausdruck des Künstlers. Kann man daher von der "Medizin als Heilkunst» sprechen? Allerdings besteht folgende Schwäche der TCM: Solche abstrakten Kunstwerke können hervorragend sein, aber in der Wissenschaft kann eine solche künstlerische Ausdruckstechnik (Deyi Wangxiang 得意忘象) nicht die objektive Entdeckung ersetzen. In der TCM hat tatsächlich diese abstrakte Struktur und ungebundene Denkart (Deyi Wangxiang) die konkrete Beschäftigung mit dem Objekt ersetzt.

\section{KARGER}

Fax +49 7614520714 Information@Karger.com Information@Kar
Prof. Dr. med. Alexander Meng

Österreichische Gesellschaft für Akupunktur c/o Tuina

Frauenfelderstrasse 8, 1170 Wien, Österreich

alexander@meng.at 
Jedes Organ hat in der TCM (藏象) eine wichtige physiologische Aufgabe, aber keines hat ein morphologisches Substrat (oder nur sehr vage, wie die Lunge, der Magen usw.). Einige Organe, wie das Mingmen 命门 (im Neijing 内经: rechts die Feuerniere/ Shenyang 肾阳 - Minghuo 命火 und links die Wasserniere/Shenyin 肾阴; oder im Nanjing 难经: links die Niere und rechts das Mingmen 命门) und die 3 Erwärmer 三焦, haben kein morphologisches Substrat [1-3].

Bock [4] meint zur modernen Medizin: «Es besteht tatsächlich kein Kontinuum des wissenschaftlichen Erkenntnisfortschrittes in der Medizin von der Antike bis in die Neuzeit, selbst wenn einzelne durchaus zutreffende Gedanken und Beobachtungen der Ärzte des früheren Zeitalters dies nahezulegen scheinen.» Etwas später liest man: «Auf jeden Fall erfolgte aber der Übergang zur heutigen wissenschaftlichen Medizin mit einem qualitativen (Sprung), der im 19. Jh. stattfand.»

\section{Paradigma der heutigen wissenschaftlichen Medizin (nach K.D. Bock)}

1. Körper und Seele des lebenden Menschen bilden eine untrennbare Einheit.

2. Das stoffliche Substrat unterliegt in seinen Elementen den Gesetzen der Physik und der Chemie.

3. Sollten zusätzlich besondere, hieraus nicht ableitbare biologische Gesetzmässigkeiten existieren, würden sie auch für den Menschen gelten.

4. Die geistig-seelischen Funktionen werden durch die Psychologie beschrieben.

5. Die Art der Beziehungen zwischen Körper und Seele (Leib-SeeleProblem) ist nur teilweise bekannt; dessen ungeachtet reagiert der Mensch in Gesundheit und Krankheit immer zugleich körperlich und seelisch.
6. Ausgehend von diesen Voraussetzungen entwickelt die medizinische Wissenschaft empirisch-rationale Verfahren zur Erkennung, Behandlung und Vorbeugung von Krankheiten.

\section{Gemeinsamkeiten von TCM und moderner Medizin}

1. Das dynamische, relative Gleichgewicht von Yin und Yang. Alle dynamischen Prozesse und alle Lebensformen können wir mit den Yin/Yang-Regeln analysieren. Die moderne Medizin analysiert aber solche Vorgänge im Einzelnen für sich.

2. Die Produktion und die Destruktion in der 5-Elemente-Lehre 五行 spiegeln das ganzheitliche Denken der TCM wider. Die 5-ElementeLehre ist ein Entsprechungssystem, welches das ganzheitliche Denken der TCM widerspiegelt. Durch die Hemmung und Aktivierung, Destruktion und Produktion wird die Wechselwirkung der Eingeweide beschrieben. Solche Wechselwirkungen der Organe kennen wir auch in der modernen Medizin, z.B. kann ein Nierenleiden Bluthochdruck auslösen. Die Modelle des Biofeedback und der Chaostheorie haben so manche Ähnlichkeiten mit der 5-Elemente-Lehre. Die TCM sieht Krankheit als Folge der gestörten Einheit des Organismus und der Beziehung Mensch-Himmel-Erde (天人合一) an.

3. Das Wechselspiel von Mensch und Umwelt ist ein weiteres Beispiel. Das Klima, die Jahreszeit, die Sternenkonstellation, die Geographie usw. haben einen intensiven Einfluss auf unser Gedeihen und Wohlbefinden.

4. Das Blackbox-Verfahren ist die Methodik der TCM. Diese erfasst durch subtilste indirekte Untersuchung, Beobachtung und Befragung (Blackbox-Verfahren) den
Mangel an Anatomie sowie den Unterschied in der Diagnose/Therapie zwischen der modernen $\mathrm{Me}-$ dizin und der TCM.

Die Informationen am Äusseren des Körpers bezeichnet Hou Can 侯灿, ein Pathophysiologe vom Zhongshan Medical College in der chinesischen Provinz Guangdon, als «intrinsic information» und «interaction information», die mittels «trial and error»Verfahren in der TCM zu ihrer speziellen Physiologie und Pathophysiologie wurden [5]. Die moderne Medizin kann z.B. aufgrund der exakten Ätiologie den Erreger mittels Antibiotika eliminieren; dass diese auch negative, den Körper schädigende Nebenwirkungen haben können, wird in Kauf genommen. Auch die exakte Substitution (Vitamin, Spurenelemente usw.) ist eine Stärke der modernen Medizin. Der Therapieerfolg wird in der modernen Medizin mit «hard data» gemessen, während die «soft data» über den Leidensdruck oft vernachlässigt werden. Die TCM legt grossen Wert auf die «clinical individuality» und die «individualized therapy» bzw. den «individualized bodily state of response». Die TCM therapiert nicht durch die Eliminierung des Erregers, weil diese nicht genau erforscht ist. Sie therapiert durch eine Stärkung des Organismus durch «motivational therapy». Die TCM legt seit jeher Wert auf die Verbesserung der «soft data», um die Lebensqualität zu verbessern.

\section{Grundregeln der Schmerztherapie mittels Akupunktur}

Zuvor ist immer eine klare Diagnose und Indikationsstellung der modernen Medizin notwendig. Das Meridiansystem als ein Regulationsund Verbindungssystem ist die wichtigste Arbeitshypothese in der Schmerztherapie. Reize treffen auf die Rezeptoren des Organismus, die als Nervenimpulse im Nervensystem weiterverarbeitet werden. Da- 
bei haben das vegetative Nervensystem, das periphere Nervensystem, das endokrine System und das Meridiansystem eine sehr enge Wechselwirkung. Das Meridiansystem hat in der TCM die Aufgabe, als Kanalsystem für den Transport von Qi, Xue 气血, Energie und Blut und somit auch als Verbindung der Eingeweide $\mathrm{zu}$ agieren. Das Meridiansystem (Kanalsystem 经络) wird in der Subcutis bzw. zwischen den Muskeln in verschiedener Tiefe lokalisiert. Die Organlehre, 5-Elemente-Lehre, Krankheitslehre und die Differenzialdiagnose sind weitere Schwerpunkte im Konzept der TCM. Wenn die Schmerzen einen Rhythmus aufweisen, dann ist die Zuhilfenahme der Organ-Uhr 生物钟 und der chronomedizinischen Regeln 五运六气 der TCM interessant. Aus praktischen Gründen ist die Einteilung nach der Schmerzlokalisation zu bevorzugen.

\section{Viszeralschmerz}

Die Organe des Thorax, Bauchs und Beckens können Störungen aufweisen, die als Schmerzen mittels eines Kardinal- oder Begleitsymptoms vorkommen. Die TCM-Differenzialdiagnose erfolgt hauptsächlich nach der Organ- und Meridian-Zuordnung. Die Differenzierung nach den Modalitäten ergänzt die Diagnose zusätzlich.

- Der akute Viszeralschmerz weist zumeist eine Fülle-Symptomatik, rasch einsetzend und sehr heftig, auf. Die Schmerztherapie ist hier symptomatisch mit dem Ziel der raschen Schmerzlinderung. Die Zirkulation des Blutes und der Vitalenergie im Meridiansystem und im Organ normalisiert sich. Lokale Schmerzpunkte, Xi-Punkte 郄穴, He-Punkte 合穴 und LuoPunkte 络穴 kommen zur Anwendung.

- Der chronische Viszeralschmerz zeigt oft eine Leere-Symptomatik 虚证. Der Schmerz besteht schon mehr als 6 Wochen, der Schmerzcharakter ist dumpf, lästig, rezi- divierend. Hierbei ist die Differenzierung der Modalitäten sehr wichtig für einen guten Therapieerfolg. Die Wurzel der Störung soll behandelt werden. Daher kommen mehr segmentale Punkte (Zustimmungspunkt und Alarmpunkt) infrage.

- Der Viszeralschmerz als Folge der Disharmonie von Qi und Xue in den Eingeweiden: Wenn bei der Differenzialdiagnose nach Körpersäften herauskommt, dass eine Kältesymptomatik 寒证 vorherrscht, dann Ren/KG 4, Di 4; wenn aber eine Hitzesymptomatik 热证 besteht, dann die entsprechenden Ying-Punkte 水穴 der Meridiane; wenn Zeichen des Blutstaus 血疼 und der Qi-Stagnation 气滞 vorkommen, dann Di 4 und Mi/MP 6.

- Projektionsschmerz als Zeichen der Organstörung: Wir können oft positive Befunde (Hautfarbe, Druckbefund, Gelose, Myogelose, Kibler'sche Verquellung usw.) am Rücken oder vorne am Rumpf feststellen. Solche Zonen sprechen oft gut auf Akupunktur und Tuina an.

\section{Schmerzen an den Extremitäten}

Hiermit sind die Schmerzen an Weichteilen und an den Gelenken der Extremitäten gemeint. Die Differenzierung nach Meridian-Zugehörigkeit ist wichtig.

- Schmerzen in Streifenform: Meist Neuralgie, an dem entsprechenden Meridian 1-3 Punkte stechen. Für die oberen Extremitäten haben sich die Punkte Di 11, Di 4 und He 1 bewährt. Für die unteren Extremitäten haben sich die Punkte Gb 30, Ma 36, Gb 34 und $\mathrm{Bl} 40$ (Weizhong) bewährt. An diesen genannten Punkten können wir das Deqi-Gefühl 得气 gut auslösen. Sollte der Patient nicht so nadelempfindlich sein (schlecht auslösbares Deqi-Gefühl), dann können wir auch eine Reihe von Punkten von einem Meridian stechen, um so das Gefühl der Ausbreitung entlang des Meridians zu erreichen.

- Schmerz an einem Ort deutet auf eine Störung in den muskulotendinären Meridianen hin: Im Akutfall kann die lokale Nadelung die Blut-Xue und den VitalenergieQi-Stau sogar verstärken. Daher empfehlen wir die Nadelung der Fernpunkte. Nach Erreichen des Deqi-Gefühls soll sich der Patient aktiv selbst mobilisieren. Wenn lokal noch Verspannung besteht, dann hier zart oberflächlich «punktieren, picken». Die Verwendung der Oppositionsregel ist hier angebracht. Im chronischen $\mathrm{Zu}-$ stand liegt oft eine lokale Stagnation von Blut-Xue und Vitalenergie-Qi vor, daher zusätzlich mehr lokale Punkte. Im Falle der Beteiligung mehrerer Meridiane oder sehr wechselhafter Schmerzlokalisation müssen allgemein regulierende Punkte verwendet werden. Solche allgemein regulierende Punkte sind alle distal vom Kniegelenk bzw. Ellbogengelenk zu finden.

- Gerne verwenden wir die PunkteKombinationen (obere und untere Extremität).

- Für die Gelenke: Di 4 und Le 3 (Schmerzen in Rahmen von Depression und Qi-Stagnation).

- Schmerzen in Rahmen von Disharmonie von Qi Xue 气血: Di 11 und Ma 36.

- Schmerzen in Rahmen von Muskel- und Sehnenverspannungen: Gb 34 und SJ/3E 5.

- Chronisch rebellierende Schmerzen: Du/LG 14 für Kopf, Thorax und Schultergürtel, Du/LG 4 für Bauchraum und Beckengürtel; Ren/KG 4 und Ren/KG 6 haben auf Yin eine roborierende Wirkung.

- Psychosomatische Schmerzen: Die Zustimmungspunkte von Kopf und Rücken haben eine beruhigende, anxiolytische und analgetische Wirkung. 
- Häufig verwenden wir folgende Punkte: Gb 20, Bl 10, Du/LG 24, die vier klugen Götter, Bl 15 (Zustimmungspunkt für das Herz), Bl 18 (Zustimmungspunkt für die Leber) und Bl 20 (Zustimmungspunkt für Mi/MP).

\section{Schmerzen am Rumpf}

Die Schmerzen am Rumpf werden in erster Linie ventral, dorsal und lateral eingeteilt. Ventral zuständig sind die Meridiane Magen und Dickdarm (Ma 44, Di 4, Mi/MP 6); lateral zuständig sind die Meridiane Gallenblase und Dreifacher Erwärmer (Le 3, Gb 34, SJ/3E 7); dorsal zuständig sind die Meridiane Harnblase und Dünndarm (Bl 60, Dü 6, Ni 3). Diese nach der Meridianzugehörigkeit ausgewählten Fernpunkte werden durch lokale Punkte ergänzt. Je akuter ein Schmerzgeschehen ist, umso mehr werden Fernpunkte bzw. je chronischer der Schmerz ist, umso mehr werden lokale Punkte verwendet.

\section{Schmerzen am Kopf}

Alle Störungen im Körper, sowohl lokal als auch allgemein bedingt, können Schmerzen an der Kopfregion auslösen. Die TCM-Differenzialdiagnose nach der Dreier-Regel hilft uns, die Fernpunkte (distal von Knie- und Ellbogengelenk) zu finden. Für die organbezogene Behandlung bewähren sich die Zustimmungspunkte und die Huatuo-Punkte (EX/B2 华佗 夹脊穴) am Rücken.

\section{Praxis}

Es ist immer zuerst eine Diagnose und Indikation nach den Regeln der modernen Medizin («Schulmedizin») zu erstellen. In allen Fällen sind psychisch wirksame Punkte zu verwenden. Wir empfehlen hierfür folgende Punkte: die vier klugen Götter, Du/ LG 20 (Punkt des hundertfachen Könnens und Zusammentreffens), Du/LG 24 (göttliche Halle), Gb 13 (göttliche Wurzel), He 7 (Tor der Götter), Pe/KS 6, Pe/KS 7, Bl 15 usw.

- Akupunktur (stechen/brennen 针 炎) und die der Akupunktur verwandten Techniken wie TuinaMassage, Moxibustion, Schröpfen, Guascha usw. sind Reflextherapien, um die Gleichgewichtsstörung des Organismus wieder in Ordnung $\mathrm{zu}$ bringen. Die Akupunktur ist eine Regulationstherapie. Sie ist keine Substitutionstherapie. In den meisten Fällen wird sie als adjuvante Therapie eingesetzt.

Dann setzen wir die Behandlung mit einem Standardprogramm und einer Standardnadelreiztechnik fort. $70 \%$ der Fälle sprechen gut auf die Behandlung an. Falls das Ansprechen nach 4-6 Sitzungen unzureichend ist, kommt die Anwendung der chinesischen Medizin dazu: Differenzialdiagnose nach der Dreier-Regel der Wiener Schule sowie Anwendung eines individuellen Programms und einer individuellen Reizmethode.

- Tuina (schieben und greifen 推拿) ist eine sanfte und bei richtiger Anwendung ungefährliche Massagetechnik aus China. Für Tuina gibt es viele Einsatzmöglichkeiten. Bei Sportverletzungen und allen Erkrankungen des Bewegungsapparats hat man gute Erfahrungen gemacht. Bei psychosomatischen Beschwerden wie Schlafstörungen, Reizbarkeit und Erschöpfungszuständen ist der Einsatz dieser Therapie ebenso sinnvoll wie bei internistischen Erkrankungen, z.B. Asthma bronchiale, Reizdarm oder Bluthochdruck, und in der Gynäkologie. Eine Therapie dauert zwischen 15 und $50 \mathrm{~min}$. Die Tuina-Therapie ergänzt in idealer Weise eine physikalische, physiotherapeutische und Akupunktur-Behandlung und steigert deren Effizienz. Die aktive Mitarbeit des Patienten in Form der Selbsthilfemethode ist in der TCM erwünscht.
- Die Dreier-Regel ist eine Vereinfachung der TCM-Diagnose für Akupunktur/Tuina im Westen für den Anfänger (nach Alexander Meng). Die Fragen 1 und 2 beziehen sich auf den Ort der Erkrankung. Dies ist wichtig für die Punktwahl. Die Frage 3 nach den Modalitäten bezieht sich auf Ätiologie, Art und Dynamik der Erkrankung. Dies ist wichtig für Prognose, Strategie der Behandlung, Reizart, Reiztechnik und Arzneitherapiekonzept. Die nach der Dreier-Regel vereinfachte Diagnose der TCM lautet «MERIDIAN + ORGAN + MODALITÄT».

\section{Gute Indikationen bei Schmerzen und ihren Begleitsymptomen}

- Gelenk- und Muskelschmerzen, Muskelverspannungen, Fibromyalgie;

- Tennisarm, Golferarm, SchulterArm-Syndrom, Zervikalsyndrom;

- Arthrose, Abnutzungserscheinungen an Schulter-, Knie-, Hüft-, Sprung- und Wirbelsäulengelenken;

- Hexenschuss, Rückenschmerzen, Kreuzschmerzen, Ischias;

- Halbseitenlähmung nach Schlaganfall;

- Gesichtsnervenlähmung (periphere Fazialisparese);

- Neuralgien (Trigeminusneuralgie, Zustand nach Gürtelrose);

- lokale, therapieresistente Schmerzen nach Verletzungen und Operationen, wie z.B. Zahnbehandlung;

- Morbus Sudeck, Phantomschmerzen, Stumpfschmerz, nach Gipsabnahme, nach Prellungen, Zerrungen, Verstauchungen usw.;

- Kopfschmerzen, Migräne;

- Kreislaufstörungen;

- Schlafstörungen;

- vegetative Störungen, posttraumatische Belastungsstörung, lavierte Depression, Angst, Burnout usw.; 
- Nikotinsucht (spricht gut an), Fresssucht, Übergewicht (nur in Kombination mit anderen Heilverfahren);

- Drogen-, Alkoholsucht (sprechen nicht gut an, nur bei stationären Patienten empfohlen)

- nervöser Magen, Gastritis, nervöse Darmbeschwerden, Reizkolon, nervöse Herzbeschwerden;

- essenzielle Hypertonie, Grenzwerthypertonie, Bluthochdruck mit psychosomatischem Aspekt;

- Obstipation, Reizblase, Potenzstörungen, Menstruationsstörungen;
- klimakterische Beschwerden, Geburtsvorbereitung, Schwangerschaftserbrechen, -kreuzschmerzen;

- Heuschnupfen, allergische Hauterkrankungen;

- Asthma bronchiale;

- Durchblutungsstörungen der Hände, Beine und Füsse;

- Stabilisierung und Stärkung der Körperabwehr;

- Steigerung der Leistungs- und Lebenskraft.

\section{Literatur}

1 Meng A, Bijak M, Stockenhuber D: Basishandbuch der Akupunktur. Wien, Maudrich 2010.

2 Meng A: TCM in Prävention und Therapie. Wien, Maudrich, 2011.

3 Meng A: Lehrbuch der Tuina-Therapie, ed 5. Stuttgart, Haug, 2006.

4 Bock KD: Wissenschaftliche und alternative Medizin. Heidelberg, Springer, 1993.

5 Can H: Einige Ansichten über die neuen Wege in der Synthese TCM und WM (chinesisch). Z Synthese TCM WM 中西结合杂志 1994:5.

6 《Zhenjiu Dacheng 针尒大成》, Yang Jizhou 杨继洲, 1601. Neue moderne Auflage, ed 2. China, Verlag für Volksgesundheit, 1995. 